\title{
A systematic review of the validity of patient derived xenograft (PDX) models: the implications for translational research and personalised medicine
}

\author{
Anne T Collins ${ }^{\text {Corresp., }}{ }^{1}$, Shona H Lang ${ }^{2}$ \\ 1 Department of Biology, University of York, York, United Kingdom \\ 2 QED Biomedical, York, United Kingdom \\ Corresponding Author: Anne T Collins \\ Email address: anne.collins@york.ac.uk
}

Patient-derived xenograft (PDX) models are increasingly been used in oncology drug development because they offer greater predictive value than traditional cell line models. Using novel tools to critique model validity and reliability we performed a systematic review to identify all original publications describing the derivation of PDX models of colon, prostate, breast and lung cancer. Validity was defined as the ability to recapitulate the disease of interest. The study protocol was registered with the Collaborative Approach to Meta-Analysis and Review of Animal Data from Experimental Studies (CAMARADES), http://www.den.ed.ac.uk/camarades/default.htm . Searches were performed in Embase, MEDLINE and Pubmed up to July 2017. A narrative data synthesis was performed. We identified 105 studies of model validations; 29 for breast, 29 for colon, 25 for lung, 23 for prostate and 4 for multiple tissues. 133 studies were excluded because they did not perform any validation experiments despite deriving a PDX. Only one study reported following the ARRIVE guidelines; developed to improve the standard of reporting for animal experimentation. Remarkably, half of all breast (52\%) and prostate (50\%) studies were judged to havehigh concern, in contrast to $16 \%$ of colon and $28 \%$ of lung studies. The validation criteria that most commonly failed (evidence to the contrary) were: tissue of origin not proven and histology of the xenograft not comparable to the parental tumour. Overall, most studies were categorized as unclear because one or more validation conditions were not reported, or researchers failed to provide data for a proportion of their models. For example, failure to demonstrate tissue of origin, response to standard of care agents and to exclude development of lymphoma. Validation tools have the potential to improve reproducibility, reduce waste in research and increase the success of translational studies. 
1 A systematic review of the validity of patient derived xenograft (PDX) models: the

2 implications for translational research and personalised medicine

3

4 Anne T Collins ${ }^{1}$, Shona H Lang ${ }^{2}$

5

$6 \quad{ }^{1}$ Department of Biology, University of York, York, UK, YO10 5DD

$7 \quad{ }^{2}$ QED Biomedical, York, UK

8

9 Corresponding author

$10 \quad$ Anne Collins ${ }^{1}$

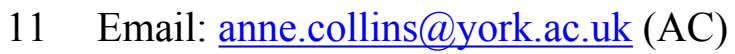

12 


\section{Abstract}

Patient-derived xenograft (PDX) models are increasingly been used in oncology drug

development because they offer greater predictive value than traditional cell line models. Using

novel tools to critique model validity and reliability we performed a systematic review to identify

17 all original publications describing the derivation of PDX models of colon, prostate, breast and

lung cancer. Validity was defined as the ability to recapitulate the disease of interest. The study

protocol was registered with the Collaborative Approach to Meta-Analysis and Review of

Animal Data from Experimental Studies (CAMARADES),

21 http://www.dcn.ed.ac.uk/camarades/default.htm. Searches were performed in Embase,

MEDLINE and Pubmed up to July 2017. A narrative data synthesis was performed. We

identified 105 studies of model validations; 29 for breast, 29 for colon, 25 for lung, 23 for

prostate and 4 for multiple tissues. 133 studies were excluded because they did not perform any

validation experiments despite deriving a PDX. Only one study reported following the ARRIVE

guidelines; developed to improve the standard of reporting for animal experimentation.

Remarkably, half of all breast (52\%) and prostate (50\%) studies were judged to have high

concern, in contrast to $16 \%$ of colon and $28 \%$ of lung studies. The validation criteria that most

commonly failed (evidence to the contrary) were: tissue of origin not proven and histology of the

xenograft not comparable to the parental tumour. Overall, most studies were categorized as

unclear because one or more validation conditions were not reported, or researchers failed to

32 provide data for a proportion of their models. For example, failure to demonstrate tissue of

33 origin, response to standard of care agents and to exclude development of lymphoma.

34 Validation tools have the potential to improve reproducibility, reduce waste in research and

35 increase the success of translational studies. 


\section{Introduction}

38 Advancing a candidate drug from preclinical testing into phase II clinical trials assumes that

39 cancer models used in the laboratory are clinically predictive. Yet, over $90 \%$ of new drugs are

40 ineffective in humans (Johnson et al., 2001; Ellis \& Fidler, 2010) suggesting that traditional

41 preclinical models, such as cell lines cultivated in monolayer or xenografts derived from them,

42 are a major factor in the low success rate of oncology drug development. A key consideration is

43 the length of time these models have been in culture, undergoing extensive adaptation and

44 selection and as such are unlikely to represent the heterogeneity and complexity of the disease.

45 In contrast, patient-derived xenograft (PDX) models, based on direct implantation of fresh cancer

46 tissue specimens from individual patients into immunodeficient mice, are reported as more

47 reliable models for preclinical research in many types of cancer (Garber, 2009; Siolas \& Hannon,

48 2013). PDXs have been cited, in numerous studies, as better predictors of response; retaining

49 cellular heterogeneity, architecture and molecular characteristic of the original cancer (Garber,

50 2009, Tentler et al., 2012). Nevertheless, there are challenges in using PDXs. For example,

51 there are inconsistencies in take rates across tumour types, and importantly tumour grades,

52 raising the question of whether PDXs are reflective of all cancer populations. Variability in take

53 rate is also associated with mouse strain (Ohbo et al., 1996). The more immune-compromised

54 strains appear to have more favourable take rates, but this is offset by the increased risk of

55 lymphoma development (Chen et al., 2012; John et al., 2012; Wetterauer et al., 2015, Taurozzi et

56 al., 2019); an under reported phenomenon in PDX research.

57 We sought to objectively assess the validity and reliability of PDX models as a platform for 58 preclinical research in the four most common cancers: breast, prostate, colon and lung. Existing 
59 risk of bias tools do not interrogate how appropriate model selection is, nor how valid the models

60 are. We previously developed novel tools to assess the validity of models, markers and the

61 imprecision of results (Collins, Ross \& Lang, 2017). This review concentrates on the assessment

62 of the scientific quality of the studies, i.e. how well the models recapitulate the disease of

63 interest, rather than the findings.

\section{Methods}

The methods for the literature searches and systematic review adhered to the Cochrane

67 Collaboration guidance (Higgins \& Green, 2011), to reduce the risk of bias and error. This study

68 was reported according to the Preferred Reporting Items for Systematic Reviews and Meta-

69 Analyses (PRISMA) statement (Moher et al., 2009), summarised in Table S1. The study protocol

70 was registered with the Collaborative Approach to Meta-Analysis and Review of Animal Data

71 from Experimental Studies (CAMARADES), http://www.den.ed.ac.uk/camarades/default.htm.

\section{Literature searches}

Attempts were made to identify studies of PDX models of breast, colon, lung and prostate carcinoma. Searches in bibliographic databases were not limited by publication date, language or publication status (published or unpublished). Search strategies are presented in Table S2. The following databases were searched on 12 July 2017: Embase (OvidSP): 1974 - 2017/07/11, Medline (OvidSP): 1946 - 2017/06/WK5, Medline In-Process Citations \& Daily Update (OvidSP): up to 2017/07/11, PubMed (NLM) (Internet) (http://www.ncbi.nlm.nih.gov/pubmed): 
80 up to $2017 / 07 / 03$. The methods section of all included articles and relevant reviews were also

81 searched to identify studies for inclusion. The searches were performed by the authors.

82

\section{Inclusion and exclusion criteria}

Inclusion and exclusion criteria are summarised in Table S2. We included original

publications, which derived and validated PDX mouse models of human breast, colon, lung and

prostate carcinoma. Specifically, we included the use of human tissue fragments or the use of

primary human carcinoma cultures ( $\leq 3$ passages) to generate xenografts in mice. Xenografts of

any passage number were considered for inclusion. At least one validation assessment question

had to be answered by the authors for inclusion; summarised in TableS3. We excluded

xenografts generated from metastatic tissue, cell lines or those established in rats. Human cells,

91 which had been genetically manipulated before xenograft generation, were excluded. PDX

92 models that were purchased or validated elsewhere were excluded. Non-English language

93 articles, conference proceedings, abstracts, commentaries and reviews were not included.

94 Publications, which included primary and metastatic samples, were included and the primary

95 samples alone were extracted where possible.

97 Study selection, data extraction and data synthesis

Publications were loaded onto the systematic review web app, Rayyan, for title and two reviewers. Articles meeting the inclusion criteria were obtained as full paper copies. Those were independently examined, in detail, by two reviewers to determine whether the full papers 
102 met the inclusion criteria of the review. All papers excluded at this second stage of the screening

103 process were documented along with the reasons for exclusion. Any discrepancies between

104 reviewers were resolved through consensus. Data extraction was performed by one reviewer and

105 checked by a second reviewer. Any discrepancies were resolved through discussion. Studies

106 were identified by the surname of the first author and by the publication year. Papers, which

107 presented validation of the same PDX model, were grouped into 'studies'. A priori outcomes for 108 extraction were primary outgrowth rate, established PDX rate and latency. During the course of

109 the review, we also decided to investigate whether the PDX models could investigate tumour

110 heterogeneity. A narrative summary of all the included studies was compiled.

\section{Quality assessment}

113 Model validity was assessed by adapting the tool created by Collins and Lang (2017) (Table S3)

114 and was defined as how well the PDX recapitulated the disease of interest. The number of PDX

115 models (or established PDX lines) derived was compared to the number of models validated. We

116 noted whether the authors had stated whether they followed the ARRIVE guidelines for the

117 reporting of animal research (Kilkenny et al., 2010). Two reviewers independently assessed

118 study quality and any discrepancies were resolved through discussion. The SYRCLE checklist

119 was not employed as it is a risk of bias tool for interventional animal studies and was not

120 appropriate (Hooijmans et al., 2014).

\section{Results}


124 Literature searches and inclusion assessment

125 A summary of the identification and selection of studies for inclusion in this review is

126 presented in Figure 1, in accordance with the PRISMA statement (Moher et al., 2009). Literature

127 searches of electronic databases retrieved 6,286 articles and hand searching identified 31

128 additional articles. After de-duplication 3640 titles/abstracts were screened and 3057 papers were

129 excluded as having no relevance to the review. Full papers of 583 potentially relevant references

130 were selected for further examination. Of these, 473 papers were excluded after reading the full

131 paper; the reasons for exclusion are provided in Figure 1 and a list of the excluded studies are

132 provided in Table S4. 133 studies were excluded because, despite deriving a PDX, the authors

133 did not perform any validation experiments. Lack of validation was defined as failure to provide

134 evidence on: a. tissue of origin, b. confirmation that the PDX was derived from a given patient,

135 c. cell lineage, d. confirmation that the PDX was derived from tumour and not normal cells, e.

136 absence of murine overgrowth, f. comparable histopathology, g. concordance for standard of care

137 agents and h. absence of lymphoma (Table S3). 110 records met the inclusion criteria; 29 for

138 breast, 29 for colon, 25 for lung, 23 for prostate and 4 for multiple tissues. Some records

139 provided validation methods for the same models, such records were grouped into 'studies'. The

140 four records for multiple tissues provided additional information for each tissue. Overall, we

141 identified 105 studies of model validations; 29 for breast, 31 for colon, 25 for lung, 20 for

142 prostate. A list of the references for the included publications and the overall studies is provided

143 in Table S5.

144

145 Characteristics of PDX models 
146 The characteristics of the PDX models are summarised in Table 1 . The majority of studies

147 reported on model development, whilst $10-42 \%$ reported the use of a PDX model to answer a

148 biomedical research question (predominantly cancer research or drug discovery). A variety of

149 mouse strains were used for derivation; the most common ( $>10 \%$ studies) were NSG,

$150 \mathrm{NOD} / \mathrm{SCID}, \mathrm{SCID}$ and Balb/c nude. The engraftment site varied according to tissue. Breast

151 models were predominantly orthotopic (55\%) or subcutaneous $(38 \%)$, whereas the majority of

152 colon and lung models were derived from subcutaneous engraftment (94\% and 75\%

153 respectively). The most common engraftment site for the derivation of prostate models was

154 subcutaneous (55\%) followed by subrenal (25\%). Most models were derived from the

155 engraftment of tissue fragments $(69 \%$ to $87 \%)$ rather than isolated cells or primary cultures. In

156 four studies reporting of methods was inadequate to ascertain whether tissue fragments or cells

157 were used.

158

159

Model validity

160

A model validity tool was previously created (Collins \& Lang, 2017), and extended to

161 include further judgements specifically for the PDX models (Table S3). Only one study (Cottu

162 et al., 2012) reported that they had followed the ARRIVE guidelines (Kilkenny et al., 2010).

163 Figure 2 summarises the overall judgements on the validity of the models. No study fully

164 validated their reported models. This would require all signalling questions to be judged at low

165 risk of concern. In breast and prostate, approximately half of the studies were judged to have

166 high concern for model validity with the remainder judged as unclear. In contrast, only $16 \%$ and

$16728 \%$ of colon and lung studies, respectively, had high concern. Overall most studies were rated 
168 as unclear; this judgment was based on a lack of information on one or more of the validation

169 questions, but without high risk concerns.

170 The first five signalling questions of the model validity tool concentrate on how well the authors

171 report methodology and sourcing of materials (Fig. S1). Such information is necessary to enable

172 others to replicate and verify findings. The majority of studies provided ethical statements for

173 the use of animal and human tissue; only $4 \%$ to $14 \%$ of studies did not. Similarly, most studies

174 reported on source and strain of mice; only $6 \%$ of breast and $10 \%$ of colon studies did not. A

175 clear description of how the mice and the xenografts were routinely maintained was not provided

176 for $24 \%$ to $48 \%$ of all studies (dependent on tissue). All studies provided a description of how

177 the PDX models were derived.

178 The second set of signalling questions judge how well the authors validated their models

179 (Fig. 3 shows judgments per tissue type and Fig S2 shows overall judgements). Individual

180 prostate study data is presented in Table S3, to allow the reader to understand the judgements.

181 Overall, most answers to any question were judged to be unclear, indicating that the authors

182 either did not investigate the question(s) or only provided data for a proportion of their models.

183 Indeed, more than half of all studies did not confirm tissue of origin, the presence of lymphoma

184 or concordance of the model with the donor sample, whilst just over half of all studies (57\%)

185 confirmed that the xenografts were derived from tumour and not normal cells (Fig. S2). Colon

186 had the fewest studies that were judged high risk. In contrast, at least one breast study had a high

187 risk of concern for each validation question (Fig. 3). Analysis of the individual signalling

188 questions indicated that for most studies there was a concern that the tissue of origin was not

189 proven. In effect, $48 \%$ of breast studies, $16 \%$ of lung and $35 \%$ of prostate were classed as high

190 risk because the models failed the authors own validation criteria for this question e.g. the 
191 xenografts did not express the stipulated tissue-specific markers. Likewise, most studies did not

192 confirm that the PDX was derived from a given patient and 3 to $17 \%$ of all studies were judged

193 as high risk because of the lack of concordant gene mutations or discordant clustering from gene

194 expression studies. Most studies did not confirm that the PDX represented the cell type of

195 interest (e.g. epithelial or neuroendocrine) and were classed as unclear, whilst 3 to $31 \%$ of

196 studies had a high risk of concern as the PDX failed the authors own validation criteria. Most

197 studies confirmed the tumorigenic nature of the PDX (32 to 66\%) whereas a high risk of concern

198 was found in $4 \%$ of breast studies, $13 \%$ colon and $8 \%$ lung. The majority of studies ( 29 to $60 \%$ )

199 confirmed that human cells were present in the xenograft; murine overgrowth can occur with

200 continuous passage (Taurozzi et al., 2017). In contrast, there was a lack of confirmation that the

201 histology of the donor tissue matched the corresponding PDX. This judgement was most often

202 made because the authors failed to provide evidence for all the models or failed to report the

203 methodology. The majority of studies did not validate whether the PDX replicated the patient

204 response to standard of care treatment. This was largely due to a lack of reported data for this

205 criterion, which is surprising given that PDXs are often reported as mimicking treatment

206 response (Garber, 2009; Tentler et al., 2012; Siolas \& Hannon, 2013). Moreover, 10\% of prostate

207 studies, 7\% breast and 4\% lung were considered high risk because of a lack of concordance with

208 patient response.

209 Lastly, we assessed whether authors validated their models for the development of

210 Epstein-Barr Virus (EBV)-associated lymphomas. We found that the majority (84\%) of studies

211 had not, therefore the risk was judged to be unclear overall. Only 15\% of studies acknowledged

212 if a PDX was not a carcinoma. Due to the heterogeneity between studies and the low number of

213 studies reporting this occurrence we have summarised the results for each study and report the 
214 range of lymphoma development over the 4 tissue types (Table 2). For breast and prostate the

215 frequency ranged from $2-80 \%$ whilst for colon the frequency ranged from 2-38\% of biopsies.

216 Although a range of mouse strains was used there was no indication that the rate of lymphoma

217 was higher with the more immunocompromised strains. One study (McAuliffe et al., 2015) was

218 judged high risk because the authors did not acknowledge a potential lymphoma and did not

219 investigate further.

220

221 Do authors validate all PDX Models?

222 We next assessed the proportion of PDX models from each study that were validated.

223 This appraisal was based on whether the author attempted to answer one or more of the

224 validation questions for all the models they derived. The results, summarised in Figure 4, show

225 that the majority of studies validated all reported models. Nevertheless, $36 \%$ of colon studies,

$22610 \%$ of prostate, $17 \%$ of breast and $28 \%$ of lung studies did not validate all published models.

227 Examination of those studies with incomplete validation (listed in Table S6) indicate that 17 of

22824 studies validated less than half of all derived PDX. For a small percentage of studies, it was

229 unclear how many models were validated as the reporting was insufficient. One study was

230 unable to derive a prostate PDX and this is reported as 'not applicable' (Fujii et al., 2008). These

231 results indicate that users cannot assume that a published PDX model has been validated.

232

233 Take rates of primary and established PDX models

234 We next considered the rates of primary and stable (e.g. PDX capable of serial transplantation)

235 outgrowth from each tissue type. Take rate is either reported as the number of xenografts formed 
236 per patient or per sample. Reporting of take rate based on the number of samples (per patient)

237 was the most difficult to assess because some studies engrafted multiple samples per mouse

238 (most often found with subrenal engraftment) or multiple mice. If clearly reported by the

239 authors we included both rates in Tables S7 and S8. Due to the heterogeneity between studies

240 (e.g. method of engraftment, strain of mouse, donor pathology and definition of outcomes) we

241 have summarised the range of take rates for each tissue type (Table 3). Excluded from this table

242 are samples from metastases and studies at high risk for model validity.

243 The range of rates for primary outgrowths from breast cancer samples was 10 to $31.3 \%$, based on

244 four studies classed as unclear for risk of bias (a further 7 reported this outcome but were judged

245 to be high risk. The remaining breast studies were not included because the authors did not report

246 this outcome). The rates reported for colon, lung and prostate xenografts were equally variable

247 due to the heterogeneity between studies and lack of reporting for this outcome. Of the colon

248 studies, 16 did not report primary outgrowth, only one was judged as high risk and the remaining

24912 studies reported primary outgrowths between 14 and $100 \%$. We considered 5 lung studies (17

250 did not report primary outgrowth and the remaining 3 were judged high risk) and 7 prostate

251 studies ( 7 were judged to be high risk and 9 did not report primary outgrowth rates).

252 As indicated in Table S7 each study defined primary outgrowth differently, this included;

253 exponential growth, any growth from implantation, tumours reaching a specific size, growth over

254 a specified time (the latter was most often used with subrenal implantation). There was similar

255 heterogeneity for the definition of stable growth which ranged from ability to passage at least 256 once in vivo to $>\mathrm{P} 5$. 
257 Some studies reported both primary and stable take rate, but this was not common; breast $\mathrm{n}=4$,

258 colon $n=5$, lung $n=3$ and prostate $n=3$. A comparison of the rates within studies showed that

259 fewer stable lines were produced overall (Table 3).

260 Individual studies attempted to investigate the effects of changing the PDX methods. One lung

261 study (Russo et al., 2015) found increased outgrowths from squamous carcinoma (92 - 96\%) in

262 comparison to adenocarcinoma (29-33\%), different outgrowth rates were also reported

263 according to hormone status and BRCA status of the donor (Zhang et al., 2013; Risbridger et al.,

264 2015). Different outgrowth rates were reported between the engraftment of tissue fragments

265 (50\%) compared to isolated cells (12.5\%; Roife et al., 2017). Such individual studies clearly

266 indicate the importance of considering all these factors in the rate of outgrowth.

267

\section{Latency of primary outgrowths}

Due to the heterogeneity between studies, we have summarised reported latencies from studies at low and unclear risk of bias for model validity (Table 4). The remaining studies either did not report latency or terminated the mice at a specific time irrespective of whether a tumour was palpable or not. Overall, colon (up to 223 days) reported the shortest latencies followed by breast (up to 279 days), lung (up to 310 days), whilst prostate had the longest latencies (up to 1147 days).

\section{Tumour heterogeneity}

277 We determined how many studies, developing PDX models from prostate cancer biopsies, had considered tumour heterogeneity by establishing multiple models from single patients, and 
279 whether all were validated. From the 20 prostate studies only $2(10 \%)$ developed multiple PDX

280 per patient and performed validation studies on all (Toivanen et al., 2011; Risbridger et al.,

281 2015). However, neither study produced stable lines from the multiple PDX. Five further studies

282 developed multiple models per patient but did not validate all of them (Wang et al., 2005; Priolo

283 et al., 2010; Chen et al., 2013; Lin et al., 2014; Lawrence et al., 2015). In summary, the majority

284 of prostate studies did not derive multiple PDX per patient nor did they clearly report this

285 information.

286

287 Discussion

288 One of the most serious obstacles confronting investigators involved in drug development is the 289 failure of existing murine tumour models to reliably predict anticancer activity in the clinic. We 290 assessed the evidence that PDX models more accurately reflect their human tumour counterpart 291 by scrutinizing models based on a checklist of strict criteria. The major finding of this review 292 was that half of all studies using prostate and breast PDX models were classed as high risk 293 because they failed (evidence to the contrary) one or more of the validation questions, for a 294 proportion of their models. We found, mostly, discordance between a PDX and corresponding 295 donor tissue for expression of tissue-specific markers, cell-specific markers and histopathology, 296 demonstrating that some PDX models may not be what they claim to be. Overall, most studies 297 were categorized as unclear because one or more validation conditions were not reported, or 298 researchers failed to provide data for a proportion of their models. The most common reasons 299 that validity was unclear were; failure to demonstrate the tissue of origin, response to standard of 300 care agents and exclusion of lymphoma. 
301 This review highlights deficiencies in reporting. For example, 133 studies were excluded because

302 the authors either failed to perform any validation experiments or simply did not report their

303 findings. Whilst the majority of included studies validated all their models, to some extent, a

304 number of published models were not validated. A lack of detail of methodology and vague

305 reporting of results made it sometimes impossible to scrutinize evidence, all of which points to a

306 failure of the peer review process. The ARRIVE guidelines, originally published in PLOS

307 Biology (Kilkenny et al., 2010), were developed in consultation with the scientific community as

308 part of an NC3Rs (National Centre for the Replacement Refinement \& Reduction of Animals in

309 Research) initiative to improve the standard of reporting of research using animals. Only one

310 study stated that they followed the guidelines (Cottu et al., 2012), yet over 1,000 journals

311 worldwide have endorsed them. It was not the aim of this review to closely assess whether

312 authors presented data in accordance with the guidelines, but it is noteworthy that $14 \%$ of studies

313 failed to provide an ethical statement and between $24-48 \%$ of studies did not provide a clear

314 description of the routine maintenance of mice before or after xeno-transplantation.

315 The tool presented here provides an 'ideal set of validation criteria' for PDX models and can be

316 adapted and applied to other models or marker studies. It may be unreasonable for a research

317 group to provide evidence to fulfil all criteria, e.g. proving the tissue of origin of an

318 undifferentiated (neuroendocrine) PDX is not straightforward. However, further studies should

319 be undertaken to prove that the PDX matches the donor tumour. Similarly, studies that use

320 primary outgrowth as their end-point (Toivanen et al., 2011; Lawrence et al., 2015; Risbridger et

321 1., 2015), particularly for drug testing, must be able to demonstrate targeting of malignant cells as

322 normal cells can also populate grafts. Indeed, our assessment of primary and stable take rates

323 show that up to $50 \%$ of primary outgrowths will not serially transplant. 
$32433 \%$ of included studies reported the use of PDX models to investigate a research question in

325 cancer research or drug discovery, highlighting the importance of rigorous validation of

326 preclinical models. Through conducting this research, we aimed to highlight issues that may help

327 alleviate the reproducibility crisis (Baker et al., 2013; Ball et al., 2015) and aid clinical

328 translation. The use of systematic reviews highlights areas of weakness that can be improved

329 going forward, but also provides a formal, unbiased and robust evaluation to provide guidance of

330 the best evidence, or as in this review, the model that best fulfils a specific research need.

331 An important consideration for the use of PDX models in cancer research is tumour

332 heterogeneity. Cancer, in an individual, is not a single disease. Tumours are heterogeneous that

333 have evolved through a process of clonal expansion and genetic diversification, ultimately

334 causing different prognoses within the same patient. The challenge for scientists and clinicians is

335 to better understand this heterogeneity at a basic biological level and determine which subclones

336 are of greatest risk to the patient (Greaves \& Maley, 2012; Bedard et al., 2013; Meacham \&

337 Morrison, 2013). For PDX models it is therefore important to establish multiple models from

338 each donor (with each clearly validated). Currently, the use of multiple models from individual

339 patients is limited, particularly so in the prostate. More aggressive tumours are easier to

340 propagate and are therefore over represented. It remains unclear why there is this selection bias

341 for more aggressive tumours or indeed why prostate cancer is underrepresented. A consensus on

342 methodology would help, but factors intrinsic to the sample are difficult to control for, such as

343 uncertainty on the amount of viable tumour being engrafted. We were unable to perform a meta-

344 analysis or funnel plot analysis of bias due to the high degree of heterogeneity between studies,

345 but long latencies reported for prostate may be one of the reasons it is under-represented. 
346 One of the major criticisms of PDX research is the lack of clear outcome definitions. Authors did

347 not clearly define engraftment rate or experimental end-point. Some reported engraftment rate as

348 the relative number of primary outgrowths or stable outgrowths (the latter defined as the ability

349 to serially passage at least once). It was also unclear if rates were based on patient numbers or

350 the number of samples. There was ambiguity on author's definition of 'successful' primary

351 outgrowth. Success was based on tumour size or growth after a specific time interval. Such

352 differences influence not only the reviewers' ability to synthesise the data but also the integrity

353 of the data (Brown et al., 2016). Clearly, 'any growth' does not provide specificity particularly if

354 that research has a clinical goal. PDX researchers should aim to have a set of agreed outcome

355 definitions to improve the field; given that there is a EurOPDX consortium (http://europdx.eu/) it

356 would be an important goal to implement.

357 We identified three other systematic reviews of PDX models; one followed PRISMA guidance

358 (Brown et al., 2016) and two did not follow PRSIMA (Jin et al., 2010; Lopez-Barcons, 2010)

359 Brown et. al. (2016) reported on the large amount of heterogeneity in engraftment rates. None of

360 the systematic reviews formally presented any quality assessment of the primary studies;

361 although SYRCLE was referenced by Brown et al (2016). All the reviews listed the validation

362 techniques reported by the primary papers, but not whether these validation techniques were

363 adequate or successful.

364 The search strategy had to be limited to the terms surrounding 'PDX'. However, it is likely that

365 if it was expanded to including broader terms, such as 'explant' then we may have found

366 additional relevant studies. Scoping searches indicated that broad terms would retrieve over 20,

367000 articles and was judged to be unfeasible. Whilst screening full papers for inclusion it was

368 difficult to assess whether the same model had been used in different reports. Poor 
369 methodological reporting and a lack of a definitive name for the model prevented the reviewer

370 from establishing multiple reports of a given PDX. This was felt to be especially the case if a

371 PDX had been licenced to a company, who had likely re-named it and not reported its derivation.

372 Similarly, we felt that the large number of studies which were excluded for not presenting any

373 validation was, in part, due to poor reporting techniques and potentially the model had been

374 validated but the results were just not reported.

375 Conclusion

376 This is the first systematic review of PDX models to provide a comprehensive assessment of

377 their validity using a novel tool (Collins \& Lang, 2017), to assess quality based on empirical

378 evidence. This is a major step forward as, until now, systematic reviews of biological models

379 have provided a subjective assessment of key components of studies that the reviewers consider

380 important (Jin et al., 2010; Lopez-Barcons, 2010; Brown et al., 2016) which does not allow a

381 scrutiny of their worth. Existing tools critique the study design and are more appropriate to

382 intervention studies (Hooijmans et al., 2014).

383 The use of systematic reviews to judge the reliability and validity of biomedical research will

384 improve the success and reproducibility of subsequent translational clinical studies, particularly

385 in this era of personalised medicine. Like similar evidence-based tools, this model validity

386 checklist represents a dynamic document and is open to improvement. We invite others to

387 comment on the tool and suggest improvements for the future. 
1. Baker M. 2016. Is there a reproducibility crisis? A Nature survey lifts the lid on how researchers view the'crisis rocking science and what they think will help. Nature 533:452-455.Ball P. The trouble with scientists. Nautil us, May. wiki.lib.sun.ac.za; 2015;14. Available: http://wiki.lib.sun.ac.za/images/d/d7/Trouble-with-scientists.pdf

2. Ball P. The trouble with scientists. Nautil us, May. wiki.lib.sun.ac.za; 2015;14. Available: http://wiki.lib.sun.ac.za/images/d/d7/Trouble-with-scientists.pdf

3. Bedard PL., Hansen AR., Ratain MJ., Siu LL. 2013. Tumour heterogeneity in the clinic. Nature 501:355-364. DOI: 10.1038/nature12627.

4. Brown KM., Xue A., Mittal A., Samra JS., Smith R., Hugh TJ. 2016. Patient-derived xenograft models of colorectal cancer in pre-clinical research: a systematic review. Oncotarget 7:66212-66225. DOI: 10.18632/oncotarget.11184.

401

402

403

404

405

406

407

408

409

410

411

412

413

414

415

416

417

418

419

420

421

422
5. Chen K., Ahmed S., Adeyi O., Dick JE., Ghanekar A. 2012. Human solid tumor xenografts in immunodeficient mice are vulnerable to lymphomagenesis associated with Epstein-Barr virus. PloS one 7:e39294. DOI: 10.1371/journal.pone.0039294.

6. Chen X., Liu B., Li Q., Honorio S., Liu X., Liu C., Multani AS., Calhoun-Davis T., Tang DG. 2013. Dissociated primary human prostate cancer cells coinjected with the immortalized Hs5 bone marrow stromal cells generate undifferentiated tumors in NOD/SCID- $\gamma$ mice. PloS one 8:e56903. DOI: 10.1371/journal.pone.0056903.

7. Collins A., Ross J., Lang SH. 2017. A systematic review of the asymmetric inheritance of cellular organelles in eukaryotes: A critique of basic science validity and imprecision. PloS one 12:e0178645. DOI: 10.1371/journal.pone.0178645.

8. Cottu P, Marangoni E, Assayag F, de Cremoux P, Vincent-Salomon A, Guyader C, de Plater L, Elbaz C, Karboul N, Fontaine JJ, Chateau-Joubert S, Boudou-Rouquette P, Alran S, Dangles-Marie V, Gentien D, Poupon M-F, Decaudin D. 2012. Modeling of response to endocrine therapy in a panel of human luminal breast cancer xenografts. Breast cancer research and treatment 133:595-606.

9. Ellis LM., Fidler IJ. 2010. Finding the tumor copycat. Therapy fails, patients don't. Nature medicine 16:974-975. DOI: 10.1038/nm0910-974.

10. EurOPDX 2018; Available: http://europdx.eu/

11. Fujii E., Suzuki M., Matsubara K., Watanabe M., Chen YJ., Adachi K., Ohnishi Y., Tanigawa M., Tsuchiya M., Tamaoki N. 2008. Establishment and characterization of in vivo human tumor models in the NOD/SCID/ $/$ cnull mouse. Pathology international 58:559-567. 
423

424

425

426

427

428

429

430

431

432

433

434

435

436

437

438

439

440

441

442

443

444

445

446

447

448

449

450

451

452

453

454

455

456

457

458

459

460
12. Garber K. 2009. From Human to Mouse and Back: "Tumorgraft" Models Surge in Popularity. Journal of the National Cancer Institute 101:6-8. DOI: 10.1093/jnci/djn481.

13. Greaves M., Maley CC. 2012. Clonal evolution in cancer. Nature 481:306-313. DOI: 10.1038/nature10762.

14. Higgins JPT, Green S (editors). Cochrane Handbook for Systematic Reviews of Interventions Version 5.1.0 [updated March 2011]. The Cochrane Collaboration, 2011. Available from www.handbook.cochrane.org.

15. Hooijmans CR., Rovers MM., de Vries RBM., Leenaars M., Ritskes-Hoitinga M., Langendam MW. 2014. SYRCLE's risk of bias tool for animal studies. BMC medical research methodology 14:43. DOI: 10.1186/1471-2288-14-43.

16. Jin K., Teng L., Shen Y., He K., Xu Z., Li G. 2010. Patient-derived human tumour tissue xenografts in immunodeficient mice: a systematic review. Clinical \& translational oncology: official publication of the Federation of Spanish Oncology Societies and of the National Cancer Institute of Mexico 12:473-480. DOI: 10.1007/s12094-010-0540-6.

17. John T., Yanagawa N., Kohler D., Craddock KJ., Bandarchi-Chamkhaleh B., Pintilie M., Sykes J., To C., Li M., Panchal D., Chen W., Shepherd FA., Tsao M-S. 2012. Characterization of Lymphomas Developing in Immunodeficient Mice Implanted With Primary Human Non-Small Cell Lung Cancer. Journal of thoracic oncology: official publication of the International Association for the Study of Lung Cancer 7:1101-1108. DOI: 10.1097/JTO.0b013e3182519d4d.

18. Johnson JI., Decker S., Zaharevitz D., Rubinstein LV., Venditti JM., Schepartz S., Kalyandrug S., Christian M., Arbuck S., Hollingshead M., Sausville EA. 2001.

Relationships between drug activity in NCI preclinical in vitro and in vivo models and early clinical trials. British journal of cancer 84:1424-1431. DOI:

10.1054/bjoc.2001.1796.

19. Kilkenny C, Browne W, Cuthill I, Emerson M, Altman D. 2014. Improving Bioscience Research Reporting: The ARRIVE Guidelines for Reporting Animal Research. Animals. Multidisciplinary Digital Publishing Institute 4:35-44. DOI:10.3390/ani4010035.

20. Lawrence MG., Pook DW., Wang H., Porter LH., Frydenberg M., Kourambas J., Appu S., Poole C., Beardsley EK., Ryan A., Others. 2015. Establishment of primary patientderived xenografts of palliative TURP specimens to study castrate-resistant prostate cancer. The Prostate 75:1475-1483.

21. Lin D., Wyatt AW., Xue H., Wang Y., Dong X., Haegert A., Wu R., Brahmbhatt S., Mo F., Jong L., Bell RH., Anderson S., Hurtado-Coll A., Fazli L., Sharma M., Beltran H., Rubin M., Cox M., Gout PW., Morris J., Goldenberg L., Volik SV., Gleave ME., Collins CC., Wang Y. 2014. High fidelity patient-derived xenografts for accelerating prostate cancer discovery and drug development. Cancer research 74:1272-1283. DOI: 10.1158/0008-5472.CAN-13-2921-T. 
461

462

463

464

465

466

467

468

469

470

471

472

473

474

475

476

477

478

479

480

481

482

483

484

485

486

487

488

489

490

491

492

493

494

495

496
22. Lopez-Barcons LA. 2010. Human prostate cancer heterotransplants: a review on this experimental model. Asian journal of andrology 12:509-518. DOI: 10.1038/aja.2010.17.

23. Meacham CE., Morrison SJ. 2013. Tumour heterogeneity and cancer cell plasticity. Nature 501:328-337. DOI: 10.1038/nature12624.

24. McAuliffe PF., Evans KW., Akcakanat A., Chen K., Zheng X., Zhao H., Eterovic AK., Sangai T., Holder AM., Sharma C., Chen H., Do K-A., Tarco E., Gagea M., Naff KA., Sahin A., Multani AS., Black DM., Mittendorf EA., Bedrosian I., Mills GB., GonzalezAngulo AM., Meric-Bernstam F. 2016. Correction: Ability to Generate Patient-Derived Breast Cancer Xenografts Is Enhanced in Chemoresistant Disease and Predicts Poor Patient Outcomes. PloS one 11:e0151121. DOI: 10.1371/journal.pone.0151121.

25. Moher D., Liberati A., Tetzlaff J., Altman DG. 2009. PRISMA Group. Preferred reporting items for systematic reviews and meta-analyses: the PRISMA statement. PLoS Med 6: e1000097. doi:10.1371/journal.pmed.1000097.

26. Ohbo K., Suda T., Hashiyama M., Mantani A., Ikebe M., Miyakawa K., Moriyama M., Nakamura M., Katsuki M., Takahashi K., Yamamura K., Sugamura K. 1996. Modulation of hematopoiesis in mice with a truncated mutant of the interleukin-2 receptor gamma chain. Blood 87:956-967.

27. Ouzzani M., Hammady H., Fedorowicz Z., Elmagarmid A. 2016. Rayyan —a web and mobile app for systematic reviews. Systematic reviews 5:210.

28. Priolo C., Agostini M., Vena N., Ligon AH., Fiorentino M., Shin E., Farsetti A., Pontecorvi A., Sicinska E., Loda M. 2010. Establishment and genomic characterization of mouse xenografts of human primary prostate tumors. The American journal of pathology 176:1901-1913. DOI: 10.2353/ajpath.2010.090873.

29. Risbridger GP., Taylor RA., Clouston D., Sliwinski A., Thorne H., Hunter S., Li J., Mitchell G., Murphy D., Frydenberg M., Pook D., Pedersen J., Toivanen R., Wang H., Papargiris M., Lawrence MG., Bolton DM. 2015. Patient-derived xenografts reveal that intraductal carcinoma of the prostate is a prominent pathology in BRCA2 mutation carriers with prostate cancer and correlates with poor prognosis. European urology 67:496-503. DOI: 10.1016/j.eururo.2014.08.007.

30. Roife D., Kang Y 'an., Wang L., Fang B., Swisher SG., Gershenwald JE., Pretzsch S., Dinney CP., Katz MHG., Fleming JB. 2017. Generation of patient-derived xenografts from fine needle aspirates or core needle biopsy. Surgery 161:1246-1254. DOI: 10.1016/j.surg.2016.11.020.

31. Russo MV., Faversani A., Gatti S., Ricca D., Del Gobbo A., Ferrero S., Palleschi A., Vaira V., Bosari S. 2015. A new mouse avatar model of non-small cell lung cancer. Frontiers in oncology 5:52. DOI: 10.3389/fonc.2015.00052. 
497

498

499

500

501

502

503

504

505

506

507

508

509

510

511

512

513

514

515

516

517

518

519

520

521

522

523

524

525

526

527

528
32. Siolas D., Hannon GJ. 2013. Patient-derived tumor xenografts: transforming clinical samples into mouse models. Cancer research 73:5315-5319. DOI: 10.1158/00085472.CAN-13-1069.

33. Taurozzi AJ., Beekharry R., Wantoch M., Labarthe M-C., Walker HF., Seed RI., Simms M., Rodrigues G., Bradford J., van der Horst G., van der Pluijm G., Collins AT. 2017. Spontaneous development of Epstein-Barr Virus associated human lymphomas in a prostate cancer xenograft program. PloS one 12:e188228. DOI:

10.1371/journal.pone.0188228.

34. Tentler JJ., Tan AC., Weekes CD., Jimeno A., Leong S., Pitts TM., Arcaroli JJ., Messersmith WA., Eckhardt SG. 2012. Patient-derived tumour xenografts as models for oncology drug development. Nature reviews. Clinical oncology 9:338-350. DOI: 10.1038/nrclinonc.2012.61.

35. Toivanen R., Berman DM., Wang H., Pedersen J., Frydenberg M., Meeker AK., Ellem SJ., Risbridger GP., Taylor RA. 2011. Brief report: a bioassay to identify primary human prostate cancer repopulating cells. Stem cells 29:1310-1314. DOI: 10.1002/stem.668.

36. Wang Y., Xue H., Cutz J-C., Bayani J., Mawji NR., Chen WG., Goetz LJ., Hayward SW., Sadar MD., Gilks CB., Gout PW., Squire JA., Cunha GR., Wang Y-Z. 2005. An orthotopic metastatic prostate cancer model in SCID mice via grafting of a transplantable human prostate tumor line. Laboratory investigation; a journal of technical methods and pathology 85:1392-1404. DOI: 10.1038/labinvest.3700335.

37. Wetterauer C., Vlajnic T., Schüler J., Gsponer JR., Thalmann GN., Cecchini M., Schneider J., Zellweger T., Pueschel H., Bachmann A., Ruiz C., Dirnhofer S., Bubendorf L., Rentsch C. 2015. Early development of human lymphomas in a prostate cancer xenograft program using triple knock-out Immunocompromised mice. The Prostate 75:585-592.

38. Zhang X., Claerhout S., Prat A., Dobrolecki LE., Petrovic I., Lai Q., Landis MD., Wiechmann L., Schiff R., Giuliano M., Wong H., Fuqua SW., Contreras A., Gutierrez C., Huang J., Mao S., Pavlick AC., Froehlich AM., Wu M-F., Tsimelzon A., Hilsenbeck SG., Chen ES., Zuloaga P., Shaw CA., Rimawi MF., Perou CM., Mills GB., Chang JC., Lewis MT. 2013. A renewable tissue resource of phenotypically stable, biologically and ethnically diverse, patient-derived human breast cancer xenograft models. Cancer research 73:4885-4897. DOI: 10.1158/0008-5472.CAN-12-4081. 
Figure 1

PRISMA flow diagram of the study selection process. 


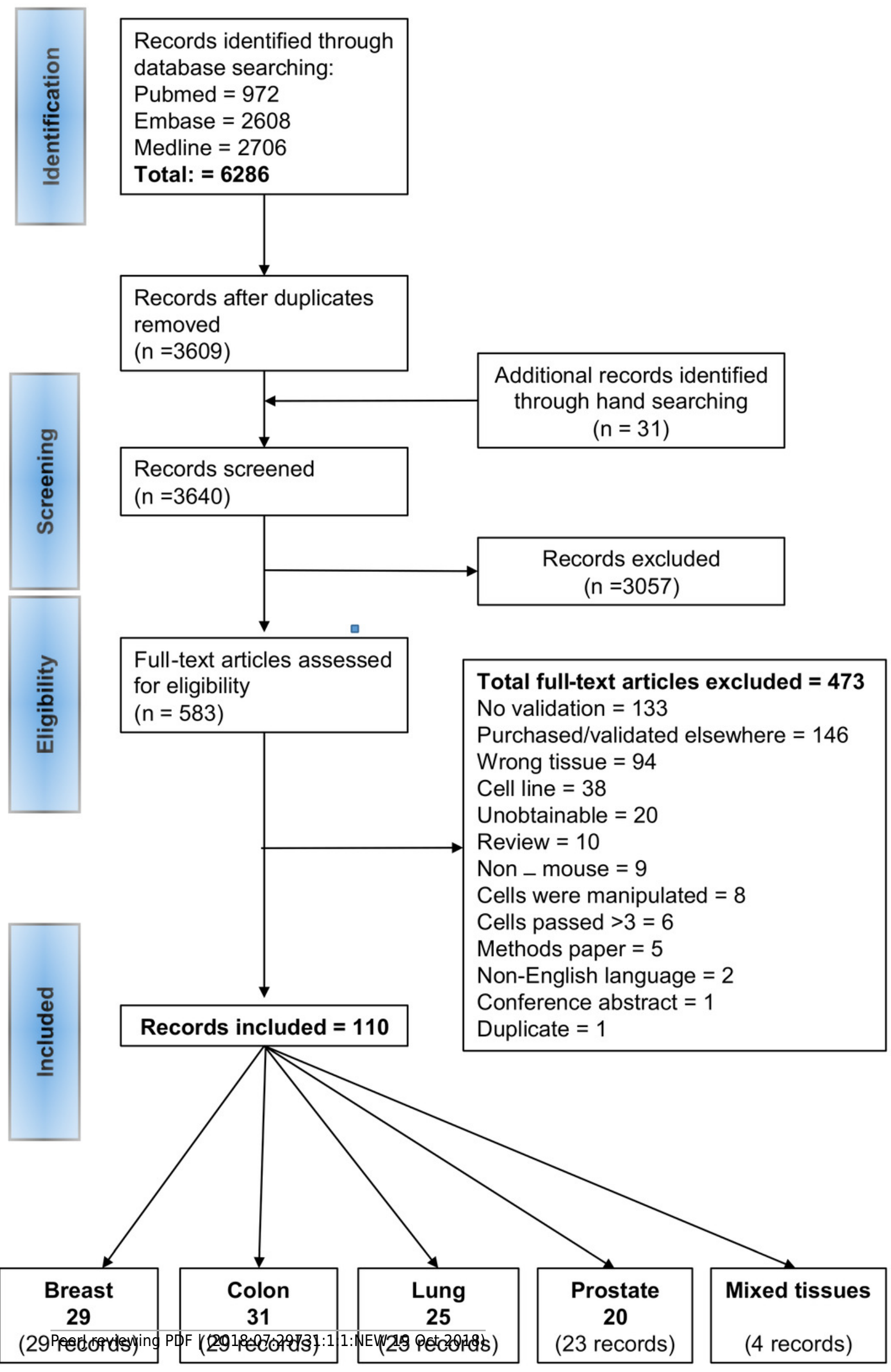




\section{Figure 2}

Overall validity ratings of PDX models.

Each study was assessed to determine if the reported PDX models were at high risk of concern for model validity. The graph indicates the percentage of studies per tissue: which had no concerns (white bars), high levels of concern (black bars), unclear levels (grey bars). $5 \%$ of prostate studies were not validated because they failed to derive a PDX.

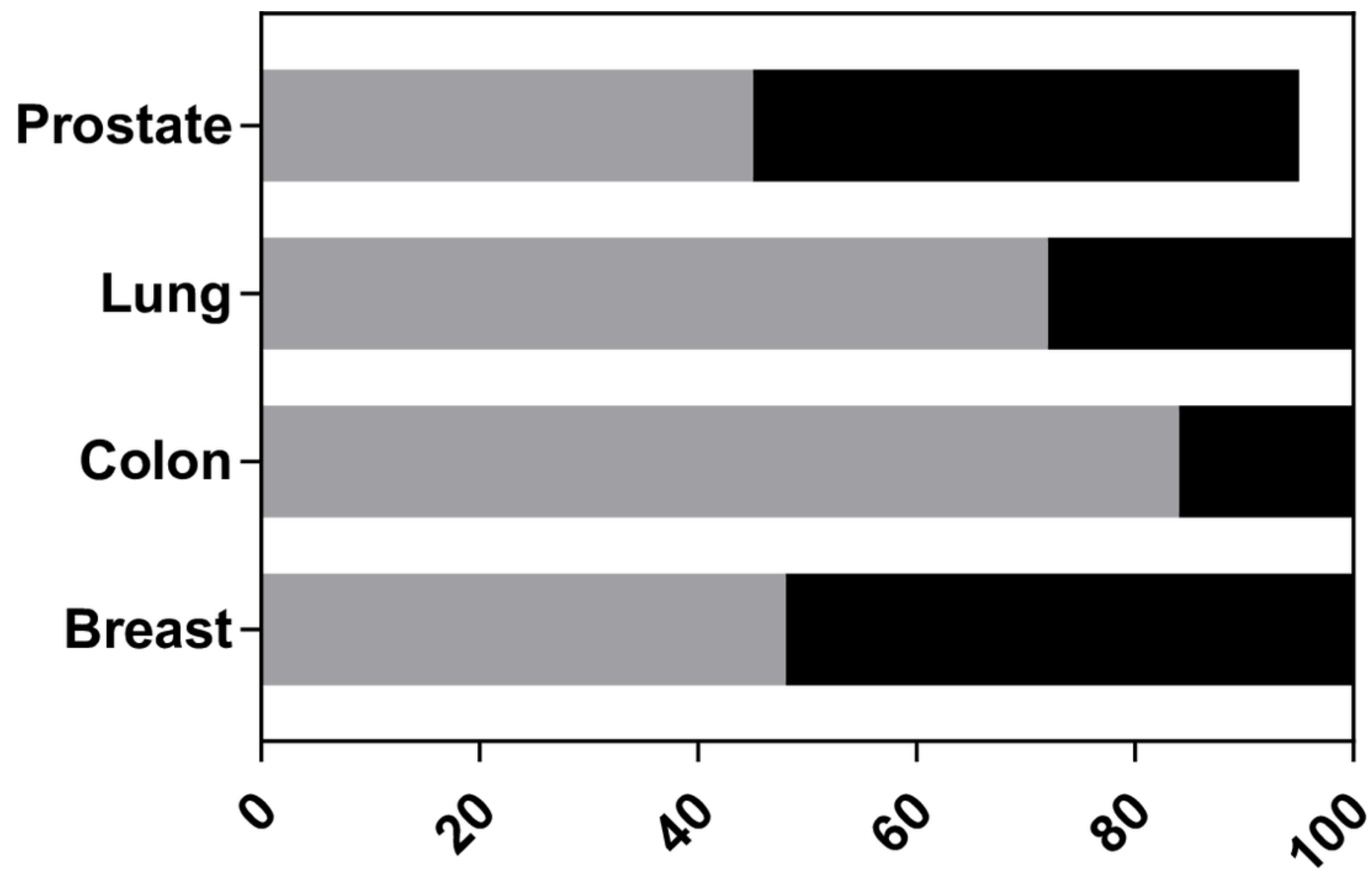




\section{Figure 3}

Overall validity ratings of PDX models.

Each study was assessed to determine the level of concern for each signalling question 8 criterion: a. was tissue of origin proven? b. confirmation that the PDX was derived from a given patient, c. was the cell lineage proven? d. confirmation that the PDX was derived from tumour and not normal cells, e. absence of murine overgrowth, $\mathrm{f}$. was there comparable histopathology? g. concordance for standard of care agents? h. was the absence of lymphoma proven? A full description of signalling questions $8 \mathrm{a}-8 \mathrm{~h}$ can also be found in supplementary Table $\mathrm{S} 3$ of the model validation tool. Each graph indicates the percentage of studies that were judged to be of low concern (white bars), high concern (black bars), unclear concern (grey bars). Breast ( $n=29$ studies). Colon ( $n=31$ studies). Lung ( $n=25$ studies). Prostate ( $n=20$ studies). 
Breast

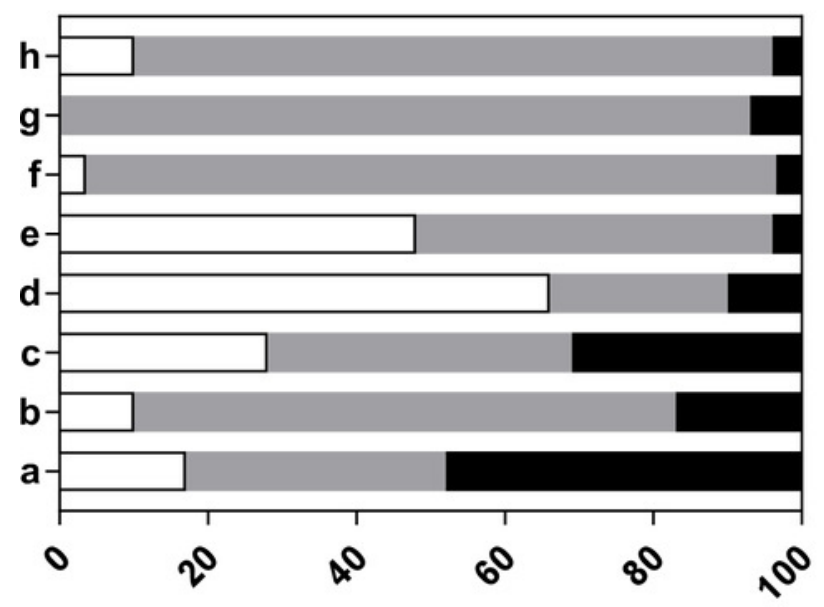

Lung

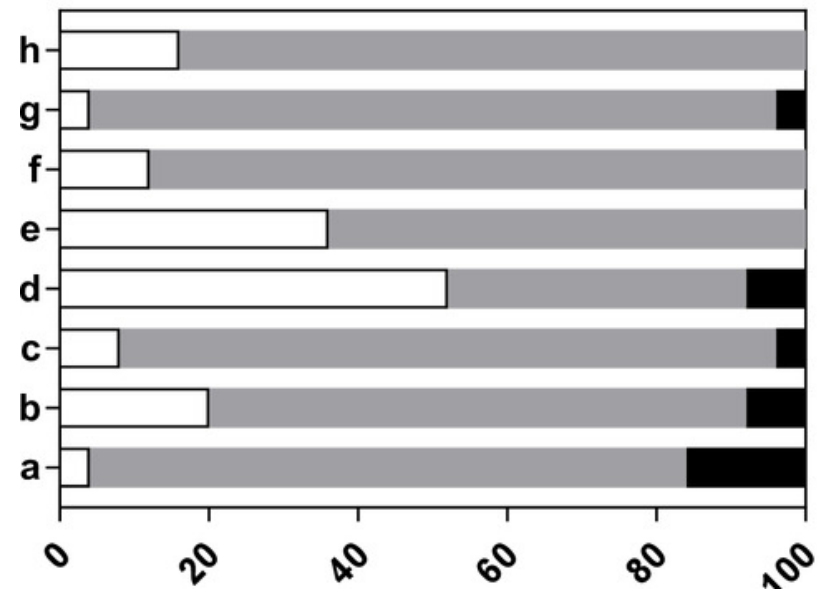

Colon

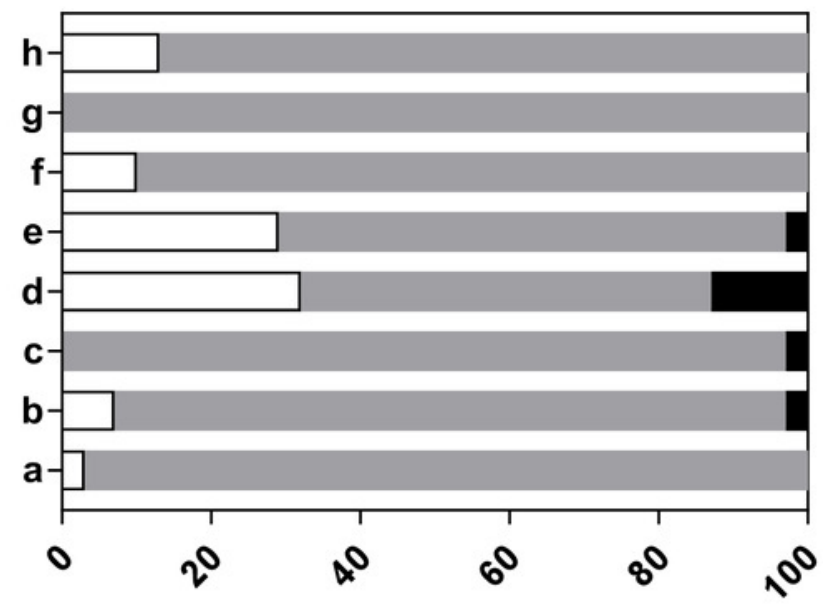

Prostate

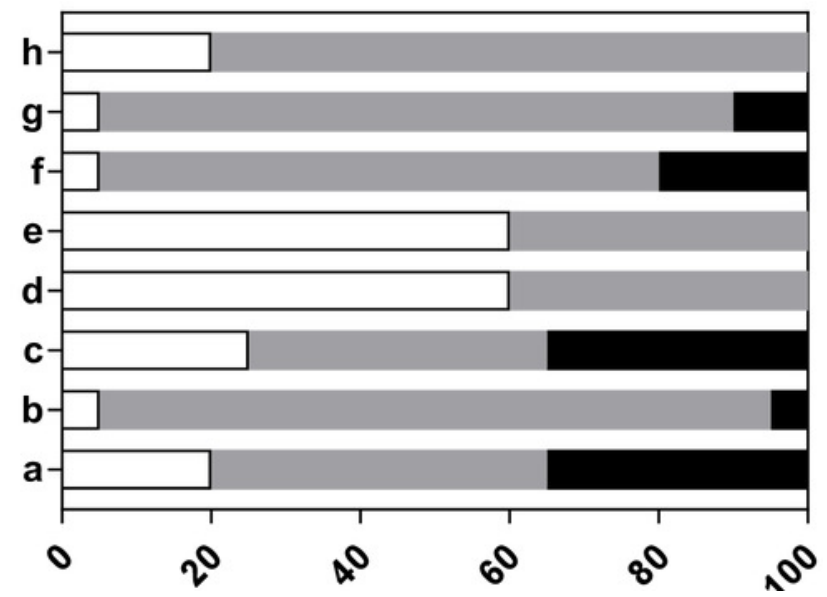




\section{Figure 4}

The proportion of PDX models from included studies that were validated .

Each study was assessed to determine if all reported PDX models were validated (any question 8 criterion). For each tissue type the percentage of studies is reported as: all models were validated (white bars), did not validate all reported models (black bars) or insufficient details reported to determine if all models were validated/not applicable (grey bars).

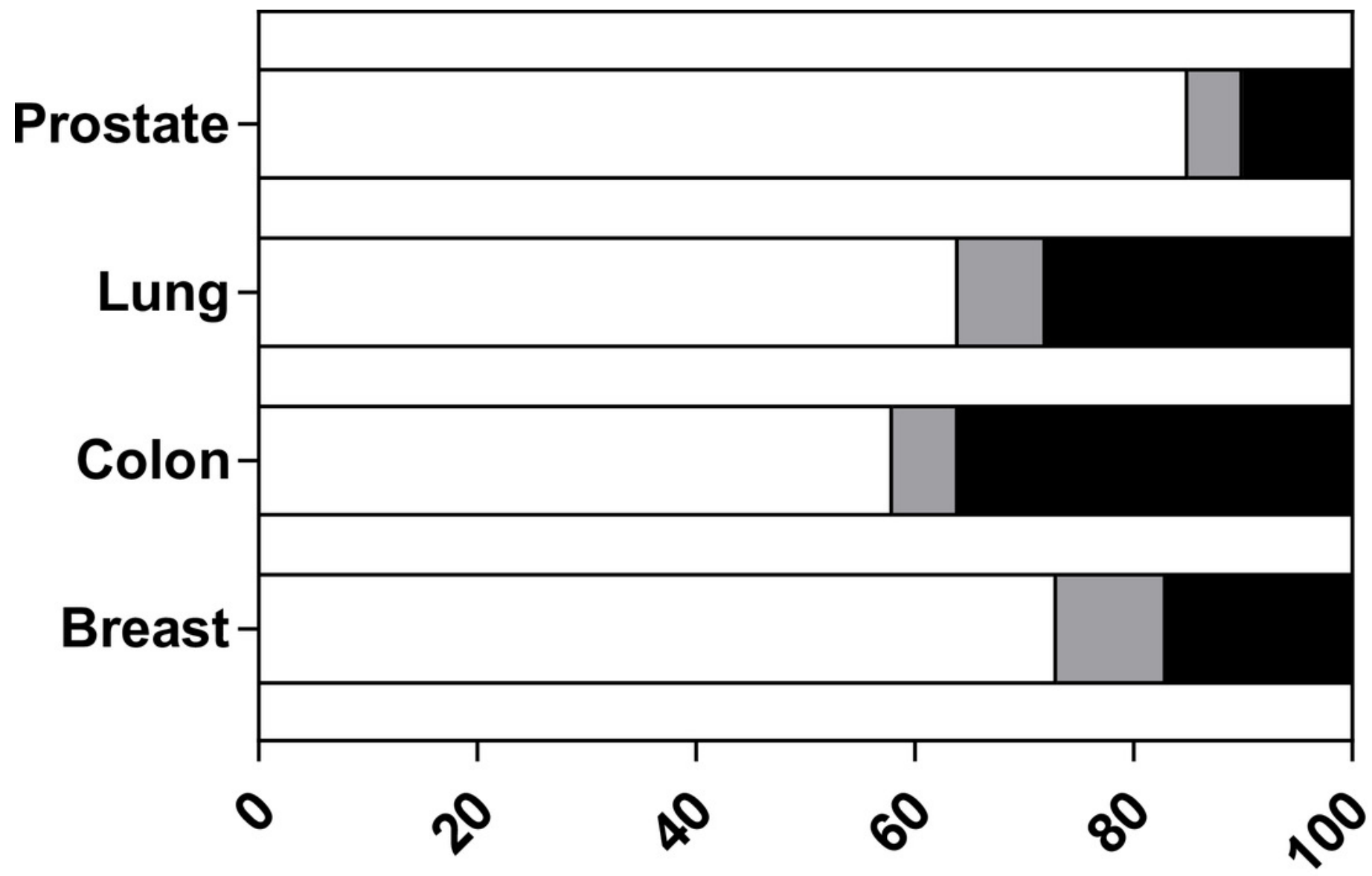




\section{Table 1 (on next page)}

Characteristics of PDX Models

The number of studies reported is shown (percentage of total tissue studies). 


\begin{tabular}{|c|c|c|c|c|}
\hline Tissue (Total studies) & $\begin{array}{l}\text { Breast } \\
(n=29)\end{array}$ & $\begin{array}{l}\text { Colorectal } \\
(n=31)\end{array}$ & $\begin{array}{l}\text { Lung } \\
\text { ( } n=25)\end{array}$ & $\begin{array}{l}\text { Prostate } \\
(n=20)\end{array}$ \\
\hline $\begin{array}{l}\text { Mouse Model: No. studies (\%) } \\
\text { B6D2F1 } \\
\text { Balb/c nude } \\
\text { Balb/c nude; SCID } \\
\text { Balb/c nude; NOD/SCID } \\
\text { SCID } \\
\text { SCID; NOD/SCID } \\
\text { CD1 nude } \\
\text { CD1 nude; SCID } \\
\text { NCG } \\
\text { NMRI nude } \\
\text { NOD/SCID } \\
\text { NOD/SCID; NMRI nude } \\
\text { NOD/SCID; NSG } \\
\text { NOD/SCID; Rag2; NSG } \\
\text { NOG } \\
\text { NSG } \\
\text { NSG; NOG } \\
\text { NSG; NRG } \\
\text { SCID/Bg; NGS } \\
\text { Swiss nude }\end{array}$ & $\begin{array}{l}1(3) \\
4(14) \\
1(3) \\
0 \\
2(7) \\
1(3) \\
0 \\
0 \\
0 \\
1(3) \\
6(21) \\
0 \\
1(3) \\
0 \\
0 \\
9(31) \\
0 \\
1(3) \\
1(3) \\
0\end{array}$ & $\begin{array}{l}1(3) \\
11(35) \\
0 \\
1(3) \\
2(7) \\
0 \\
0 \\
0 \\
0 \\
1(3) \\
7(23) \\
0 \\
1(3) \\
0 \\
1(3) \\
4(13) \\
0 \\
0 \\
0 \\
2(7)\end{array}$ & $\begin{array}{l}1(4) \\
4(16) \\
0 \\
0 \\
2(8) \\
0 \\
2(8) \\
1(4) \\
1(4) \\
0 \\
10(40) \\
1(4) \\
0 \\
0 \\
1(4) \\
2(8) \\
0 \\
0 \\
0 \\
0\end{array}$ & $\begin{array}{l}0 \\
4(20) \\
0 \\
1(5) \\
6(30) \\
0 \\
0 \\
0 \\
0 \\
1(5) \\
3(15) \\
0 \\
1(5) \\
1(5) \\
0 \\
2(10) \\
1(5) \\
0 \\
0 \\
0\end{array}$ \\
\hline $\begin{array}{l}\text { Engraftment of cells or tissue: } \\
\text { isolated cells } \\
\text { tissue fragments } \\
\text { isolated cells, tissue fragments } \\
\text { primary culture } \\
\text { minced tissue } \\
\text { unclear }\end{array}$ & $\begin{array}{l}3(10) \\
20(69) \\
2(7) \\
1(3) \\
0 \\
3(10)\end{array}$ & $\begin{array}{l}2(7) \\
27(87) \\
0 \\
0 \\
1(3) \\
1(3)\end{array}$ & $\begin{array}{l}2(8) \\
21(84) \\
1(4) \\
1(4) \\
0 \\
0\end{array}$ & $\begin{array}{l}0 \\
17(85) \\
3(15) \\
0 \\
0 \\
0\end{array}$ \\
\hline $\begin{array}{l}\text { Engraftment site: } \\
\text { orthotopic } \\
\text { subcutaneous } \\
\text { subcutaneous, subrenal } \\
\text { subrenal } \\
\text { subrenal, subcutaneous, orthotopic }\end{array}$ & $\begin{array}{l}16(55) \\
11(38) \\
0 \\
1(3) \\
1(3)\end{array}$ & $\begin{array}{l}0 \\
29(94) \\
1(3) \\
1(3) \\
0\end{array}$ & $\begin{array}{l}2(8) \\
18(75) \\
0 \\
5(17) \\
0\end{array}$ & $\begin{array}{l}0 \\
11(55) \\
1(5) \\
5(25) \\
3(15)\end{array}$ \\
\hline $\begin{array}{l}\text { Use of PDX: } \\
\text { Model establishment } \\
\text { Biomarkers/ cancer research/ drug } \\
\text { discovery }\end{array}$ & $\begin{array}{l}19(66) \\
10(34)\end{array}$ & $\begin{array}{l}19(61) \\
12(39)\end{array}$ & $\begin{array}{l}16(58) \\
10(42)\end{array}$ & $\begin{array}{c}18(90) \\
2(10)\end{array}$ \\
\hline
\end{tabular}

1 Table 1: Characteristics of PDX Models 
Table 2 (on next page)

Frequency of lymphoma formation

Subcut $=$ subcutaneous; Ortho $=$ orthotopic; mets $=$ metastatic . 


\begin{tabular}{|c|c|c|c|c|c|c|}
\hline Tissue & References & Mouse model & Engraftment site & Sample origin & $\begin{array}{l}\text { No. } \\
\text { patients }\end{array}$ & $\begin{array}{l}\text { \% Biopsies } \\
\text { forming } \\
\text { Lymphomas }\end{array}$ \\
\hline Breast & $\begin{array}{l}\text { Fujii } 2008 \\
\text { Bondarenko } 2015 \\
\text { Wagasugi } 1995 \\
\text { DeRose } 2011\end{array}$ & $\begin{array}{l}\text { NSG } \\
\text { NSG } \\
\text { Balb/c nude } \\
\text { NOD/SCID }\end{array}$ & $\begin{array}{l}\text { Subcut } \\
\text { Subcut } \\
\text { Subcut } \\
\text { Ortho }\end{array}$ & $\begin{array}{l}\text { primary } \\
\text { primary + mets } \\
\text { unclear } \\
\text { primary + mets }\end{array}$ & $\begin{array}{l}57 \\
3 \\
5 \\
42\end{array}$ & $\begin{array}{l}2 \\
33 \\
80 \\
2\end{array}$ \\
\hline Colon & $\begin{array}{l}\text { Fujii } 2008 \\
\text { Bondarenko } 2015 \\
\text { Mukohyama 2016* } \\
\text { Zhang } 2015\end{array}$ & $\begin{array}{l}\text { NSG } \\
\text { NSG } \\
\text { NOD/SCID, NSG } \\
\text { NOD/SCID }\end{array}$ & $\begin{array}{l}\text { Subcut } \\
\text { Subcut } \\
\text { Subcut } \\
\text { Subcut }\end{array}$ & $\begin{array}{l}\text { primary } \\
\text { primary + mets } \\
\text { primary } \\
\text { primary }\end{array}$ & $\begin{array}{l}48 \\
7 \\
5,8 \\
43\end{array}$ & $\begin{array}{l}38 \\
28.5 \\
20,13 \\
2.3\end{array}$ \\
\hline Lung & $\begin{array}{l}\text { Anderson } 2015 \\
\text { Llie } 2015 \\
\text { Fujii } 2008 \\
\text { John } 2011\end{array}$ & $\begin{array}{l}\text { NOD/SCID } \\
\text { CD1 nude, SCID } \\
\text { NSG } \\
\text { NOD/SCID }\end{array}$ & $\begin{array}{l}\text { Ortho } \\
\text { Subcut } \\
\text { Subcut } \\
\text { Subcut }\end{array}$ & $\begin{array}{l}\text { primary } \\
\text { primary + mets } \\
\text { primary } \\
\text { primary }\end{array}$ & $\begin{array}{l}10 \\
100 \\
2 \\
157\end{array}$ & $\begin{array}{l}10 \\
15 \\
50 \\
12\end{array}$ \\
\hline Prostate & $\begin{array}{l}\text { Fujii } 2008 \\
\text { Lin } 2013 \\
\text { Wetterauer } 2015 \\
\text { Klein } 1997\end{array}$ & $\begin{array}{l}\text { NSG } \\
\text { NOD/SCID } \\
\text { NSG, NOG } \\
\text { SCID }\end{array}$ & $\begin{array}{l}\text { Subcut } \\
\text { Subrenal } \\
\text { Subcut; subrenal } \\
\text { Subcut }\end{array}$ & $\begin{array}{l}\text { primary } \\
\text { primary; primary }+ \text { mets } \\
\text { primary } \\
\text { primary }\end{array}$ & $\begin{array}{l}12 \\
16,18 \\
27 \\
3\end{array}$ & $\begin{array}{l}17 \\
12.5,11 \\
80 \\
33\end{array}$ \\
\hline
\end{tabular}

1 Table 2. Lymphoma Formation 


\section{Table 3(on next page)}

Primary Outgrowth Rate and Established PDX Outgrowth Rate

Values based on primary tumour samples. Excluded are studies with high risk of model validity. Individual level study data are reported in supplementary Tables S7 and S8. 
1 Table 3: Primary Outgrowth Rate and Established PDX Outgrowth Rate

\begin{tabular}{|c|c|c|c|}
\hline Tissue & $\begin{array}{c}\text { Range of reported } \\
\text { Primary outgrowth rates (\%) }\end{array}$ & $\begin{array}{c}\text { Range of reported } \\
\text { Established PDX rates (\%) }\end{array}$ & $\begin{array}{c}\text { Percentage of PDX forming } \\
\text { stable lines (Median) }\end{array}$ \\
\hline Breast & 10 to 30 & 5 to 27 & 55.8 \\
\hline Colon & 14 to 100 & 10 to 41 & 68 \\
\hline Lung & 26 to 90 & 9 to 83 & 71.1 \\
\hline Prostate & 0 to 100 & 0 to 33 & 67 \\
\hline
\end{tabular}




\section{Table 4(on next page)}

Latency of primary outgrowths

. $*$ Derived from a primary culture. Subcut $=$ subcutaneous; Ortho $=$ orthotopic. Mets $=$ metastatic tumours. NR $=$ sample origin not reported. 
Table 4. Latency of primary outgrowths

\begin{tabular}{|c|c|c|c|c|c|c|c|}
\hline Study & Mouse strain & $\begin{array}{c}\text { Engraftment } \\
\text { site }\end{array}$ & Sample origin & $\begin{array}{c}\text { Reported } \\
\text { mean latency } \\
\text { (days) }\end{array}$ & $\begin{array}{c}\text { Reported } \\
\text { median latency } \\
\text { (days) }\end{array}$ & $\begin{array}{c}\text { Reported } \\
\text { range (days) }\end{array}$ & $\begin{array}{l}\text { No. } \\
\text { PDX }\end{array}$ \\
\hline Mc Auliffe 2015 & Balc/c nude & subcut & Breast & 109 & & $40-217$ & 13 \\
\hline Wagasugi 1995 & Balb/c nude & subcut & unclear & & & $78-279$ & 7 \\
\hline Bogachek 2015* & Nude & subcut & Breast & 21 & & & 1 \\
\hline Davies 1981 & Nude & subcut & Colon & 35 & & & 9 \\
\hline Guan 2016 & Balb/c nude & subcut & Colon & 94 & & & 340 \\
\hline $\operatorname{Jin} 2011$ & Balb/c nude & subcut & Colon & 141 & & & 12 \\
\hline Julien 2012 & Swiss nude & subcut & Colon + Mets & & 59 & & 35 \\
\hline Zhou 2011 & Balb/c nude & subcut & NR & 21 & & $15-30$ & 3 \\
\hline Hao 2015 & NOD/SCID & subcut & Lung & 112 & & $62-310$ & 23 \\
\hline Leong 2014 & NSG & subcut & Lung & 104 & & & NR \\
\hline Zhang 2013 & SCID, nude & subcut & Lung & & & $20-95$ & 14 \\
\hline Roife 2017 & NOD/SCID & subcut & Lung & 58 & & $26-175$ & 9 \\
\hline Lin 2013 & NOD/SCID & subrenal & Prostate, +Mets & 570,485 & & $93-1147$ & 9,12 \\
\hline Yoshikawa 2016 & SCID & subcut & Prostate & 270 & & & 1 \\
\hline Pretlow 1993 & nude & subcut & Prostate & & & $60-330$ & 10 \\
\hline Klein 1997 & SCID & subcut & Prostate & 300 & & & 2 \\
\hline
\end{tabular}




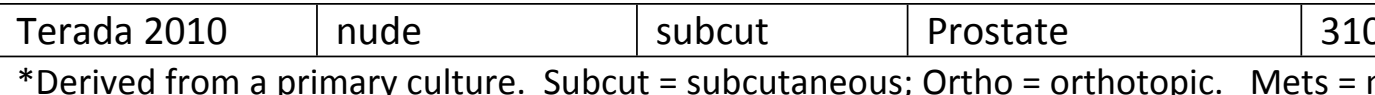

